\title{
A well posedness result for nonlinear viscoelastic equations with memory
}

\author{
Monica Conti, Elsa M. Marchini, Vittorino Pata * \\ Politecnico di Milano - Dipartimento di Matematica "F.Brioschi", Via Bonardi 9, 20133 Milano, Italy
}

Received 29 May 2013

Accepted 13 August 2013

Communicated by Enzo Mitidieri

\section{Introduction}

Given a bounded domain $\Omega \subset \mathbb{R}^{3}$ with smooth boundary $\partial \Omega$, we denote by

$$
A=-\Delta
$$

the Dirichlet operator with domain

$$
\operatorname{dom}(A)=H^{2}(\Omega) \cap H_{0}^{1}(\Omega) \Subset L^{2}(\Omega) .
$$

Let $\rho \in[0,4], \theta \in[0,1], \gamma \geq 0$ and $\alpha>0$ be fixed parameters. For $t \in \mathbb{R}^{+}=(0, \infty)$, we consider the equation

$$
\left|\partial_{t} u\right|^{\rho} \partial_{t t} u+A \partial_{t t} u+\gamma A^{\theta} \partial_{t} u+\alpha A u-\int_{0}^{\infty} \mu(s) A u(t-s) \mathrm{d} s+f(u)=h
$$

in the unknown variable $u=u(\boldsymbol{x}, t): \Omega \times \mathbb{R} \rightarrow \mathbb{R}$ subject to the Dirichlet boundary condition

$$
u(\boldsymbol{x}, t)_{\mid \boldsymbol{x} \in \partial \Omega}=0 .
$$

The model is supplemented with the initial conditions (the dependence on $\boldsymbol{x}$ is omitted)

$$
u(0)=u_{0}, \quad \partial_{t} u(0)=v_{0}, \quad u(-s)_{\mid s \in \mathbb{R}^{+}}=\psi_{0}(s),
$$

where $u_{0}, v_{0}: \Omega \rightarrow \mathbb{R}$ and $\psi_{0}: \Omega \times \mathbb{R}^{+} \rightarrow \mathbb{R}$ are given functions, the latter accounting for the initial past history of $u$.

\footnotetext{
* Corresponding author. Tel.: +39 0223994583; fax: +39 0223994621.

E-mail addresses: monica.conti@polimi.it (M. Conti), elsa.marchini@polimi.it (E.M. Marchini), vittorino.pata@polimi.it, v.pata@hotmail.it (V. Pata).
} 
Here, the time-independent external force $h$ belongs to the dual space $H^{-1}(\Omega)$ of $H_{0}^{1}(\Omega)$, while the locally Lipschitz nonlinearity $f$, with $f(0)=0$, fulfills the critical growth restriction

$$
|f(u)-f(v)| \leq c|u-v|\left(1+|u|^{4}+|v|^{4}\right),
$$

along with the dissipation condition

$$
\liminf _{|u| \rightarrow \infty} \frac{f(u)}{u}>-\lambda_{1},
$$

where $\lambda_{1}>0$ is the first eigenvalue of $A$. Finally, the convolution (or memory) kernel $\mu$ is a nonnegative, nonincreasing, piecewise absolutely continuous function on $\mathbb{R}^{+}$of total mass

$$
\int_{0}^{\infty} \mu(s) \mathrm{d} s=\kappa \in[0, \alpha) .
$$

Without loss of generality, we may take

$$
\alpha-\kappa=1 .
$$

In particular, $\mu$ is allowed to exhibit (even infinitely many) jumps, and can be unbounded about the origin.

Remark 1.1. The degenerate case $\mu \equiv 0$, corresponding to the partial differential equation

$$
\left|\partial_{t} u\right|^{\rho} \partial_{t t} u+A \partial_{t t} u+\gamma A^{\theta} \partial_{t} u+A u+f(u)=h,
$$

is included in our analysis.

Problem (1.1), featuring the nonlinear term

$$
\left|\partial_{t} u\right|^{\rho} \partial_{t t} u
$$

provides a generalization, accounting for memory effects in the material, of equations of the form

$$
\varrho\left(\partial_{t} u\right) \partial_{t t} u+A \partial_{t t} u+A u=0 .
$$

Such PDEs arise in mechanics, in the description of the vibrations of thin rods whose material density $\varrho\left(\partial_{t} u\right)$ is not constant (see e.g. [1]). The model under consideration has been the object of intensive investigations in the last decade, mainly in its simplified Volterra version

$$
\left|\partial_{t} u\right|^{\rho} \partial_{t t} u+A \partial_{t t} u+\gamma A^{\theta} \partial_{t} u+\alpha A u-\int_{0}^{t} \mu(s) A u(t-s) \mathrm{d} s=0,
$$

which turns out to be a particular instance of (1.1), as shown in the next Section 3.

The first result concerning (1.6) appears in [2], where the global existence of weak solutions is established for $\theta=1$ and $\gamma \geq 0$, provided that

$$
\rho \leq 2 .
$$

Besides, assuming $\gamma>0$ and an exponentially decaying memory kernel $\mu$, the authors demonstrate the exponential decay of solutions. However, since no uniqueness is proved, such a result holds only for those trajectories that can be obtained as limits in the Galerkin approximation scheme.

After [2], the study of the longterm properties of the (Galerkin) solutions to (1.6) has been tackled in several works (e.g. [3-12]), with various decay hypotheses on $\mu$. Still, in the above-mentioned papers, the restriction $\rho \leq 2$ is always assumed. Indeed, all of them refer to [2] for the existence result, with the only exception of [4], which actually recasts the argument of [2].

On the contrary, the uniqueness issue has never been addressed until the very recent article [13], dealing with the more general model (1.1) with $\theta=1$, in presence of a nonlinearity $f(u)$ of cubic growth satisfying $u f(u) \geq 0$. There, leaning on [2], the authors show the existence of solutions for $\rho \leq 2$, and they prove a continuous dependence (whence uniqueness) result under the additional request that the map

$$
v \mapsto|v|^{\rho}
$$

be differentiable at zero, which introduces the further restriction

$$
\rho>1 \text {. }
$$

The aim of our paper is to establish the ultimate well-posedness result for (1.1)-(1.2), ensuring existence (see Section 4) and continuous dependence from the initial data (see Section 5 ) for the most general admissible nonlinearity $f(u)$, as well as for $\rho$ belonging to the whole meaningful range $[0,4]$.

Once well-posedness is attained, the study of the asymptotic properties of the solution semigroup becomes a meaningful and interesting question, that will be possibly addressed in forthcoming papers. 


\section{Functional setting}

For $r \in \mathbb{R}$, we define the scale of compactly nested Hilbert spaces

$$
\mathrm{H}^{r}=\operatorname{dom}\left(A^{r / 2}\right)
$$

with inner products and norms given by

$$
\langle u, v\rangle_{r}=\left\langle A^{r / 2} u, A^{r / 2} v\right\rangle_{L^{2}(\Omega)} \quad \text { and } \quad\|u\|_{r}=\left\|A^{r / 2} u\right\|_{L^{2}(\Omega)} .
$$

We will always omit the index $r$ whenever $r=0$. Thus, $\mathrm{H}=L^{2}(\Omega)$. The symbol $\langle\cdot, \cdot\rangle$ will also stand for the duality product between $\mathrm{H}^{1}=H_{0}^{1}(\Omega)$ and $\mathrm{H}^{-1}=H^{-1}(\Omega)$. Then, we introduce the so-called history space

$$
\mathcal{M}=L_{\mu}^{2}\left(\mathbb{R}^{+} ; \mathrm{H}^{1}\right)
$$

endowed with the inner product

$$
\langle\eta, \xi\rangle_{\mathcal{M}}=\int_{0}^{\infty} \mu(s)\langle\eta(s), \xi(s)\rangle_{1} \mathrm{~d} s .
$$

We will also consider the right-translation semigroup $\Sigma(t)$ on $\mathcal{M}$ acting as

$$
[\Sigma(t) \eta](s)= \begin{cases}0 & 0<s \leq t, \\ \eta(s-t) & s>t\end{cases}
$$

whose infinitesimal generator is the linear operator

$$
T \eta=-\eta^{\prime}, \quad \operatorname{dom}(T)=\left\{\eta \in \mathcal{M}: \eta^{\prime} \in \mathcal{M}, \eta(0)=0\right\},
$$

the prime standing for weak derivative. The following inequality holds (see e.g. [14])

$$
\langle T \eta, \eta\rangle_{\mathcal{M}} \leq 0, \quad \forall \eta \in \operatorname{dom}(T) .
$$

Finally, we introduce the extended history space

$$
\mathscr{H}=\mathrm{H}^{1} \times \mathrm{H}^{1} \times \mathcal{M} .
$$

Notation. Throughout the paper, $c \geq 0$ and $Q(\cdot)$ will stand for a generic constant and a generic increasing positive function, respectively. We will use, often without explicit mention, the usual Sobolev embeddings, as well as the Young, Hölder and Poincaré inequalities.

\section{The equation in the history framework}

Following the approach of Dafermos [15], the original problem (1.1) translates into the system in the unknown variables $u=u(t)$ and $\eta=\eta^{t}(s)$

$$
\left\{\begin{array}{l}
\left|\partial_{t} u\right|^{\rho} \partial_{t t} u+A \partial_{t t} u+\gamma A^{\theta} \partial_{t} u+A u+\int_{0}^{\infty} \mu(s) A \eta(s) \mathrm{d} s+f(u)=h, \\
\partial_{t} \eta=T \eta+\partial_{t} u
\end{array}\right.
$$

where the initial conditions (1.2) become

$$
u(0)=u_{0}, \quad \partial_{t} u(0)=v_{0}, \quad \text { and } \quad \eta_{0}(s)=u_{0}-\psi_{0}(s) .
$$

At a formal level, this is obtained by defining the auxiliary variable

$$
\eta=\eta^{t}(\boldsymbol{x}, s): \Omega \times[0, \infty) \times \mathbb{R}^{+} \rightarrow \mathbb{R},
$$

accounting for the past history of $u$, as

$$
\eta^{t}(s)=u(t)-u(t-s) .
$$

First, we give the definition of weak solution.

Definition 3.1. Given $\tau>0$, a pair $(u, \eta)$ with

$$
u \in W^{2, \infty}\left(0, \tau ; \mathrm{H}^{1}\right) \text { and } \eta \in \mathcal{C}([0, \tau], \mathcal{M})
$$

is said to be a (weak) solution to (3.1) on the time-interval $[0, \tau]$ with initial data

$$
\left(u(0), \partial_{t} u(0), \eta^{0}\right)=\left(u_{0}, v_{0}, \eta_{0}\right) \in \mathscr{H}
$$


if for every test function $\phi \in \mathrm{H}^{1}$ the equality

$$
\left\langle\left|\partial_{t} u\right|^{\rho} \partial_{t t} u, \phi\right\rangle+\left\langle\partial_{t t} u, \phi\right\rangle_{1}+\gamma\left\langle\partial_{t} u, \phi\right\rangle_{\theta}+\langle u, \phi\rangle_{1}+\int_{0}^{\infty} \mu(s)\langle\eta(s), \phi\rangle_{1} \mathrm{~d} s+\langle f(u), \phi\rangle=\langle h, \phi\rangle
$$

holds for a.e. $t \in[0, \tau]$, and $\eta$ is a mild solution on $[0, \tau]$ in the sense of Pazy [16] to the nonhomogeneous linear equation in the Hilbert space $\mathcal{M}$

$$
\frac{\mathrm{d}}{\mathrm{d} t} \eta=T \eta+\partial_{t} u
$$

Remark 3.2. Note that the condition $u \in W^{2, \infty}\left(0, \tau ; H^{1}\right)$ implies

$$
u \in \mathcal{C}^{1}\left([0, \tau], \mathrm{H}^{1}\right) .
$$

Therefore, $\partial_{t} u$ can be viewed as an element of the space $L^{1}(0, \tau ; \mathcal{M})$. Accordingly, the mild solution $\eta$ to (3.2) belongs to $\mathcal{C}([0, \tau], \mathcal{M})($ see $[16$, Chapter 4$])$.

Remark 3.3. By definition, for $\eta$ to be a mild solution to (3.2) with initial datum $\eta^{0}=\eta_{0} \in \mathcal{M}$ is the same as saying that

$$
\eta^{t}=\Sigma(t) \eta_{0}+\int_{0}^{t} \Sigma(t-y) \partial_{t} u(y) \mathrm{d} y .
$$

This entails the explicit representation formula for $\eta$

$$
\eta^{t}(s)= \begin{cases}u(t)-u(t-s) & 0<s \leq t \\ \eta_{0}(s-t)+u(t)-u_{0} & s>t\end{cases}
$$

As a particular case of the problem above, we recover the Volterra equation

$$
\left|\partial_{t} u\right|^{\rho} \partial_{t t} u+A \partial_{t t} u+\gamma A^{\theta} \partial_{t} u+\alpha A u-\int_{0}^{t} \mu(s) A u(t-s) \mathrm{d} s+f(u)=h,
$$

where $\alpha$ fulfills (1.5). Indeed (see [17]), by choosing

$$
\eta_{0}(s)=u_{0},
$$

formula (3.3) yields

$$
\eta^{t}(s)= \begin{cases}u(t)-u(t-s) & 0<s \leq t \\ u(t) & s>t\end{cases}
$$

Accordingly,

$$
A u(t)+\int_{0}^{\infty} \mu(s) A \eta^{t}(s) \mathrm{d} s=\alpha A u(t)-\int_{0}^{t} \mu(s) A u(t-s) \mathrm{d} s .
$$

Therefore, Definition 3.1 for the Volterra equation (3.4) becomes

Definition 3.4. A function $u \in W^{2, \infty}\left(0, \tau ; H^{1}\right)$ is said to be a (weak) solution to (3.4) on $[0, \tau]$ with initial data

$$
\left(u(0), \partial_{t} u(0)\right)=\left(u_{0}, v_{0}\right) \in \mathrm{H}^{1} \times \mathrm{H}^{1}
$$

if for every test function $\phi \in \mathrm{H}^{1}$ the equality

$$
\left\langle\left|\partial_{t} u\right|^{\rho} \partial_{t t} u, \phi\right\rangle+\left\langle\partial_{t t} u, \phi\right\rangle_{1}+\gamma\left\langle\partial_{t} u, \phi\right\rangle_{\theta}+\alpha\langle u, \phi\rangle_{1}-\int_{0}^{t} \mu(s)\langle u(t-s), \phi\rangle_{1} \mathrm{~d} s+\langle f(u), \phi\rangle=\langle h, \phi\rangle
$$

holds for a.e. $t>0$.

\section{Existence of solutions}

Along the section, let $\tau>0$ be arbitrarily fixed.

Theorem 4.1. For any initial data $z=\left(u_{0}, v_{0}, \eta_{0}\right) \in \mathcal{H}$, system (3.1) admits at least a solution $\left(u(t), \eta^{t}\right)$ on $[0, \tau]$ satisfying the initial conditions

$$
\left(u(0), \partial_{t} u(0), \eta^{0}\right)=z .
$$


In particular, defining the corresponding energy at time $t$ as

$$
E(t)=\frac{1}{2}\left[\|u(t)\|_{1}^{2}+\left\|\partial_{t} u(t)\right\|_{1}^{2}+\left\|\eta^{t}\right\|_{\mathcal{M}}^{2}\right],
$$

the following proposition holds.

Proposition 4.2. For every initial data $z$ with $\|z\|_{\mathscr{H}} \leq R$ and every $t \in[0, \tau]$, we have the uniform estimate

$$
E(t)+\left\|\partial_{t t} u(t)\right\|_{1} \leq \mathbb{Q}(R),
$$

where the increasing function $\mathbb{Q}$ is independent of $\tau>0$.

The existence result follows from a standard Galerkin approximation scheme, the key point being the validity of some a priori estimates.

\subsection{Energy estimates}

Assume for the moment to have a sufficiently regular solution $(u, \eta)$ to (3.1) with initial data $\|z\|_{\mathscr{H}} \leq R$, and define the energy functional

$$
\mathfrak{L}(t)=\frac{1}{\rho+2} \int_{\Omega}\left|\partial_{t} u(t)\right|^{\rho+2} \mathrm{~d} \boldsymbol{x}+E(t)+\langle\hat{f}(u(t)), 1\rangle-\langle h, u(t)\rangle,
$$

where

$$
\hat{f}(u)=\int_{0}^{u} f(y) \mathrm{d} y .
$$

In light of assumptions (1.3)-(1.4), it is readily seen that

$$
v E-c \leq \mathfrak{L} \leq c E^{3}+c
$$

for some $v>0$, possibly very small, depending only on the value of the limit in (1.4). Testing system (3.1) with $\left(\partial_{t} u, \eta\right)$ in $\mathrm{H}$ $\times \mathcal{M}$ and recalling (2.1), we get

$$
\frac{\mathrm{d}}{\mathrm{d} t} \mathfrak{L}+\gamma\left\|\partial_{t} u\right\|_{\theta}^{2}=\langle T \eta, \eta\rangle_{\mathcal{M}} \leq 0 .
$$

Hence,

$$
\mathfrak{L}(t) \leq \mathfrak{L}(0) \leq \mathbb{Q}(R) .
$$

In light of (4.1), this yields

$$
E(t) \leq Q(R) .
$$

Furthermore, a multiplication of the first equation of (3.1) by $\partial_{t t} u$ gives

$$
\left\langle\left|\partial_{t} u\right|^{\rho} \partial_{t t} u, \partial_{t t} u\right\rangle+\left\|\partial_{t t} u\right\|_{1}^{2}=-\gamma\left\langle\partial_{t} u, \partial_{t t} u\right\rangle_{\theta}-\left\langle u, \partial_{t t} u\right\rangle_{1}-\int_{0}^{\infty} \mu(s)\left\langle\eta(s), \partial_{t t} u\right\rangle_{1} \mathrm{~d} s-\left\langle f(u), \partial_{t t} u\right\rangle+\left\langle h, \partial_{t t} u\right\rangle .
$$

The first term on the left-hand side is positive and, by (1.3),

$$
-\left\langle f(u), \partial_{t t} u\right\rangle \leq\|f(u)\|_{L^{6 / 5}}\left\|\partial_{t t} u\right\|_{L^{6}} \leq c\left(1+\|u\|_{1}^{5}\right)\left\|\partial_{t t} u\right\|_{1} .
$$

Moreover,

$$
-\int_{0}^{\infty} \mu(s)\left\langle\eta(s), \partial_{t t} u\right\rangle_{1} \mathrm{~d} s \leq\left\|\partial_{t t} u\right\|_{1} \int_{0}^{\infty} \mu(s)\|\eta(s)\|_{1} \mathrm{~d} s,
$$

where

$$
\int_{0}^{\infty} \mu(s)\|\eta(s)\|_{1} \mathrm{~d} s \leq\left(\int_{0}^{\infty} \mu(s) \mathrm{d} s\right)^{\frac{1}{2}}\left(\int_{0}^{\infty} \mu(s)\|\eta(s)\|_{1}^{2} \mathrm{~d} s\right)^{\frac{1}{2}}=\sqrt{\kappa}\|\eta\|_{\mathcal{M}} .
$$

Thus, we infer from (4.3) that

$$
\begin{aligned}
\left\|\partial_{t t} u\right\|_{1}^{2} & \leq\left(\gamma\left\|\partial_{t} u\right\|_{2 \theta-1}+\|u\|_{1}+\sqrt{\kappa}\|\eta\|_{\mathcal{M}}+c+c\|u\|_{1}^{5}+\|h\|_{-1}\right)\left\|\partial_{t t} u\right\|_{1} \\
& \leq \frac{1}{2}\left\|\partial_{t t} u\right\|_{1}^{2}+\mathcal{Q}(R),
\end{aligned}
$$

implying the further bound

$$
\left\|\partial_{t t} u(t)\right\|_{1} \leq \mathcal{Q}(R) .
$$

Incidentally, once existence will be proved, (4.3)-(4.4) establish Proposition 4.2. 


\subsection{Galerkin scheme}

We follow a standard Galerkin method based on the choice of a suitable orthonormal basis of $\mathrm{H}^{1} \times \mathcal{M}$ (see [18] for more details). We then consider the smooth solutions $\left(u_{n}, \eta_{n}\right)$ on $[0, \tau]$ to the corresponding $n$-dimensional Cauchy approximating problems with initial conditions

$$
z_{n}=\left(u_{0 n}, v_{0 n}, \eta_{0 n}\right) \rightarrow z=\left(u_{0}, v_{0}, \eta_{0}\right) \text { in } \mathcal{H} .
$$

Arguing as in the previous subsection, we deduce the bounds

$$
\left\|u_{n}(t)\right\|_{1}+\left\|\partial_{t} u_{n}(t)\right\|_{1}+\left\|\partial_{t t} u_{n}(t)\right\|_{1}+\left\|\eta_{n}^{t}\right\|_{\mathcal{M}} \leq \mathcal{Q}(R) .
$$

Therefore, we find a pair $(u, \eta)$ such that, up to subsequences,

$$
\begin{aligned}
& u_{n} \rightarrow u \quad \text { weakly- } * \text { in } L^{\infty}\left(0, \tau ; \mathrm{H}^{1}\right), \\
& \partial_{t} u_{n} \rightarrow \partial_{t} u \quad \text { weakly- } * \text { in } L^{\infty}\left(0, \tau ; \mathrm{H}^{1}\right), \\
& \partial_{t t} u_{n} \rightarrow \partial_{t t} u \text { weakly- } * \text { in } L^{\infty}\left(0, \tau ; \mathrm{H}^{1}\right), \\
& \eta_{n} \rightarrow \eta \quad \text { weakly- } * \text { in } L^{\infty}(0, \tau ; \mathcal{M}) .
\end{aligned}
$$

\subsection{Passage to the limit}

We aim to show that $(u, \eta)$ is a weak solution to (3.1), passing to the limit in the $n$-dimensional approximated problem solved by $\left(u_{n}, \eta_{n}\right)$. On account of (4.5)-(4.8), the only difficulty here is handling the nonlinear terms. To this end, calling $\Omega_{\tau}$ $=\Omega \times(0, \tau)$, we begin to observe that

$$
f\left(u_{n}\right) \text { and }\left|\partial_{t} u_{n}\right|^{\rho} \partial_{t} u_{n}
$$

are uniformly bounded in $L^{6 / 5}\left(\Omega_{\tau}\right)$. Indeed, since $\rho \in[0,4]$, exploiting the $\mathrm{H}^{1}$-bound for $\partial_{t} u_{n}$ we have

$$
\begin{aligned}
\int_{0}^{\tau} \int_{\Omega}\left|\partial_{t} u_{n}\right|^{\frac{6}{5}(\rho+1)} \mathrm{d} \boldsymbol{x} \mathrm{d} t & \leq c \int_{0}^{\tau}\left(\int_{\Omega}\left|\partial_{t} u_{n}\right|^{6} \mathrm{~d} \boldsymbol{x}\right)^{\frac{\rho+1}{5}} \mathrm{~d} t \\
& \leq c \int_{0}^{\tau}\left\|\partial_{t} u_{n}\right\|_{1}^{\frac{6(\rho+1)}{5}} \mathrm{~d} t \\
& \leq \tau Q(R),
\end{aligned}
$$

while the corresponding estimate for $f\left(u_{n}\right)$ follows in a similar fashion making use of the growth condition (1.3). Therefore, the compact embedding

$$
W^{1, \infty}\left(0, \tau ; H^{1}\right) \Subset \mathcal{C}([0, \tau], H),
$$

jointly with the $\mathrm{H}^{1}$-bounds for $\partial_{t} u_{n}$ and $\partial_{t t} u_{n}$, provide the convergence (up to a subsequence)

$$
\partial_{t} u_{n} \rightarrow \partial_{t} u \text { in } \mathcal{C}([0, \tau], \mathrm{H}) .
$$

In particular,

$$
\left|\partial_{t} u_{n}\right|^{\rho} \partial_{t} u_{n} \rightarrow\left|\partial_{t} u\right|^{\rho} \partial_{t} u \quad \text { a.e. in } \Omega_{\tau}
$$

Hence, exploiting the $L^{6 / 5}$-bound, the weak dominated convergence theorem entails the limit

$$
-\frac{1}{\rho+1} \int_{0}^{\tau}\left\langle\left|\partial_{t} u_{n}\right|^{\rho} \partial_{t} u_{n}, \partial_{t} \zeta\right\rangle \mathrm{d} t \rightarrow \int_{0}^{\tau}\left\langle\left|\partial_{t} u\right|^{\rho} \partial_{t t} u, \zeta\right\rangle \mathrm{d} t
$$

for every fixed $\zeta \in \mathscr{D}\left([0, \tau], \mathrm{H}^{1}\right)$, having used the identity

$$
\int_{0}^{\tau}\left\langle\left|\partial_{t} u\right|^{\rho} \partial_{t t} u, \zeta\right\rangle \mathrm{d} t=-\frac{1}{\rho+1} \int_{0}^{\tau}\left\langle\left|\partial_{t} u\right|^{\rho} \partial_{t} u, \partial_{t} \zeta\right\rangle \mathrm{d} t .
$$

On the other hand, as

$$
\int_{0}^{\tau}\left\langle\left|\partial_{t} u_{n}\right|^{\rho} \partial_{t t} u_{n}, \zeta\right\rangle \mathrm{d} t=-\frac{1}{\rho+1} \int_{0}^{\tau}\left\langle\left|\partial_{t} u_{n}\right|^{\rho} \partial_{t} u_{n}, \partial_{t} \zeta\right\rangle \mathrm{d} t,
$$

we also get

$$
\int_{0}^{\tau}\left\langle\left|\partial_{t} u_{n}\right|^{\rho} \partial_{t t} u_{n}, \zeta\right\rangle \mathrm{d} t \rightarrow \int_{0}^{\tau}\left\langle\left|\partial_{t} u\right|^{\rho} \partial_{t t} u, \zeta\right\rangle \mathrm{d} t
$$


Due to the arbitrariness of $\zeta$, we conclude that

$$
\left\langle\left|\partial_{t} u_{n}\right|^{\rho} \partial_{t t} u_{n}, \phi\right\rangle \rightarrow\left\langle\left|\partial_{t} u\right|^{\rho} \partial_{t t} u, \phi\right\rangle
$$

for every $\phi \in \mathrm{H}^{1}$ and a.e. $t \in[0, \tau]$. Analogously, we can pass to the limit in the second nonlinearity. Namely, by the same compact embedding above, the $\mathrm{H}^{1}$-bounds for $u_{n}$ and $\partial_{t} u_{n}$ entail the convergence (up to a subsequence)

$$
u_{n} \rightarrow u \text { a.e. in } \Omega_{\tau},
$$

and, by the continuity of $f$,

$$
f\left(u_{n}\right) \rightarrow f(u) \text { a.e. in } \Omega_{\tau} .
$$

At this point, the limit

$$
\int_{0}^{\tau}\left\langle f\left(u_{n}\right), \zeta\right\rangle \mathrm{d} t \rightarrow \int_{0}^{\tau}\langle f(u), \zeta\rangle \mathrm{d} t
$$

follows by virtue of the uniform $L^{6 / 5}$-bound for $f\left(u_{n}\right)$.

Summarizing, we proved that $(u, \eta)$ solve in the weak sense the first equation of $(3.1)$ on the time-interval $[0, \tau]$. Finally, we turn our attention to $\eta$. Since in the approximation scheme $\eta_{n} \in \operatorname{dom}(T)$, it follows that the representation formula (3.3) for $\eta_{n}$ holds, namely,

$$
\eta_{n}^{t}(s)= \begin{cases}u_{n}(t)-u_{n}(t-s) & 0<s \leq t, \\ \eta_{0 n}(s-t)+u_{n}(t)-u_{0 n} & s>t .\end{cases}
$$

At this point, knowing that $u_{0 n} \rightarrow u_{0}$ and $\eta_{0 n} \rightarrow \eta_{0}$ strongly, and recalling (4.5), we easily conclude that $\eta_{n} \rightarrow \tilde{\eta}$ in some weak topology, where

$$
\tilde{\eta}^{t}(s)= \begin{cases}u(t)-u(t-s) & 0<s \leq t, \\ \eta_{0}(s-t)+u(t)-u_{0} & s>t,\end{cases}
$$

and the convergence (4.8) forces the equality $\tilde{\eta}=\eta$. Thus, as $\eta$ fulfills (3.3), it is a mild solution to the second equation of (3.1).

\section{Continuous dependence and uniqueness}

Again, $\tau>0$ is arbitrarily fixed. Given $z_{1}, z_{2} \in \mathcal{H}$, let $\left(u_{1}, \eta_{l}\right)$, with $\imath=1,2$, be any two solutions to (3.1) on the timeinterval $[0, \tau]$ with initial data

$$
\left(u_{l}(0), \partial_{t} u_{l}(0), \eta_{l}^{0}\right)=z_{l} \text {. }
$$

Defining the difference of solutions

$$
\bar{u}=u_{1}-u_{2} \text { and } \bar{\eta}=\eta_{1}-\eta_{2} \text {, }
$$

we have the following continuous dependence estimate.

Theorem 5.1. For every $R \geq 0$, the estimate

$$
\|\bar{u}(t)\|_{1}^{2}+\left\|\partial_{t} \bar{u}(t)\right\|_{1}^{2}+\left\|\bar{\eta}^{t}\right\|_{\mathcal{M}}^{2} \leq\left(1+\tau^{3}\right) \mathcal{Q}(R) e^{\tau(1+\tau) Q(R)}\left\|z_{1}-z_{2}\right\|_{\mathscr{H}}^{2}
$$

holds for every $t \in[0, \tau]$ whenever $\left\|z_{l}\right\|_{\mathscr{H}} \leq R$. The increasing function $\mathcal{Q}$ is independent of $\tau>0$.

Choosing $z_{1}=z_{2}$, we readily draw the next corollary.

Corollary 5.2. The solution to (3.1) is unique.

Proof of Theorem 5.1. We introduce the new variables

$$
w(t)=\int_{0}^{t} u(y) \mathrm{d} y \text { and } \xi^{t}(s)=\int_{0}^{t} \eta^{y}(s) \mathrm{d} y .
$$

Defining the function

$$
\sigma(v)=\frac{1}{1+\rho} v|v|^{\rho},
$$

and integrating the first equation of (3.1) on $(0, t)$, we obtain

$$
\sigma\left(\partial_{t} u\right)+A \partial_{t t} w+\gamma A^{\theta} \partial_{t} w+A w+\int_{0}^{\infty} \mu(s) A \xi(s) \mathrm{d} s+\int_{0}^{t} f(u(y)) \mathrm{d} y=t h+g,
$$


where

$$
g=A \partial_{t} u(0)+\gamma A^{\theta} u(0)+\sigma\left(\partial_{t} u(0)\right) .
$$

Given any two solutions $\left(u_{l}, \eta_{l}\right)$ to (3.1), denote the corresponding differences by

$$
\bar{w}=w_{1}-w_{2} \text { and } \bar{\xi}=\xi_{1}-\xi_{2} .
$$

Then $(\bar{w}, \bar{\xi})$ satisfies the system

$$
\begin{aligned}
& \sigma\left(\partial_{t} u_{1}\right)-\sigma\left(\partial_{t} u_{2}\right)+A \partial_{t t} \bar{w}+\gamma A^{\theta} \partial_{t} \bar{w}+A \bar{w}+\int_{0}^{\infty} \mu(s) A \bar{\xi}(s) \mathrm{d} s+F=G, \\
& \partial_{t} \bar{\xi}=T \bar{\xi}+\partial_{t} \bar{w}-\bar{u}(0)+\bar{\eta}^{0}
\end{aligned}
$$

having set

$$
F(t)=\int_{0}^{t}\left[f\left(u_{1}(y)\right)-f\left(u_{2}(y)\right)\right] d y
$$

and

$$
G=A \partial_{t} \bar{u}(0)+\gamma A^{\theta} \bar{u}(0)+\sigma\left(\partial_{t} u_{1}(0)\right)-\sigma\left(\partial_{t} u_{2}(0)\right) .
$$

Multiplying (5.1) by $\partial_{t t} \bar{w}$, and observing that by the monotonicity of $\sigma$

$$
\left\langle\sigma\left(\partial_{t} u_{1}\right)-\sigma\left(\partial_{t} u_{2}\right), \partial_{t t} \bar{w}\right\rangle \geq 0,
$$

we obtain

$$
\left\|\partial_{t t} \bar{w}\right\|_{1}^{2} \leq-\gamma\left\langle\partial_{t} \bar{w}, \partial_{t t} \bar{w}\right\rangle_{\theta}-\left\langle\bar{w}, \partial_{t t} \bar{w}\right\rangle_{1}-\int_{0}^{\infty} \mu(s)\left\langle\bar{\xi}(s), \partial_{t t} \bar{w}\right\rangle_{1} \mathrm{~d} s-\left\langle F, \partial_{t t} \bar{w}\right\rangle+\left\langle G, \partial_{t t} \bar{w}\right\rangle .
$$

We now define the energy corresponding to the pair $(\bar{w}, \bar{\xi})$ as

$$
\Lambda(t)=\frac{1}{2}\left[\|\bar{w}(t)\|_{1}^{2}+\left\|\partial_{t} \bar{w}(t)\right\|_{1}^{2}+\left\|\bar{\xi}^{t}\right\|_{\mathcal{M}}^{2}\right] .
$$

A multiplication of (5.1) by $\partial_{t} \bar{w}$ and (5.2) by $\bar{\xi}$ in $\mathcal{M}$ gives

$$
\frac{\mathrm{d}}{\mathrm{d} t} \Lambda+\gamma\left\|\partial_{t} \bar{w}\right\|_{\theta}^{2}=\langle T \bar{\xi}, \bar{\xi}\rangle_{\mathcal{M}}-\left\langle\sigma\left(\partial_{t} u_{1}\right)-\sigma\left(\partial_{t} u_{2}\right), \partial_{t} \bar{w}\right\rangle-\left\langle F, \partial_{t} \bar{w}\right\rangle+\left\langle G, \partial_{t} \bar{w}\right\rangle+\left\langle\bar{\eta}^{0}-\bar{u}(0), \bar{\xi}\right\rangle_{\mathcal{M}} .
$$

Adding (5.3)-(5.4), with the aid of (2.1), we arrive at

$$
\begin{aligned}
\frac{\mathrm{d}}{\mathrm{d} t} \Lambda+\gamma\left\|\partial_{t} \bar{w}\right\|_{\theta}^{2}+\left\|\partial_{t t} \bar{w}\right\|_{1}^{2} \leq & -\left\langle\sigma\left(\partial_{t} u_{1}\right)-\sigma\left(\partial_{t} u_{2}\right), \partial_{t} \bar{w}\right\rangle \\
& -\frac{\mathrm{d}}{\mathrm{d} t}\left\langle F, \bar{w}+\partial_{t} \bar{w}\right\rangle+\left\langle\partial_{t} F, \bar{w}+\partial_{t} \bar{w}\right\rangle+\frac{\mathrm{d}}{\mathrm{d} t}\left\langle G, \bar{w}+\partial_{t} \bar{w}\right\rangle \\
& +\left\langle\bar{\eta}^{0}-\bar{u}(0), \bar{\xi}\right\rangle_{\mathcal{M}}-\gamma\left\langle\partial_{t} \bar{w}, \partial_{t t} \bar{w}\right\rangle_{\theta}-\left\langle\bar{w}, \partial_{t t} \bar{w}\right\rangle_{1}-\int_{0}^{\infty} \mu(s)\left\langle\bar{\xi}(s), \partial_{t t} \bar{w}\right\rangle_{1} \mathrm{~d} s .
\end{aligned}
$$

In order to control the terms on the right-hand side, we exploit the bounds

$$
\left\|u_{l}\right\|_{1}+\left\|\partial_{t} u_{l}\right\|_{1}+\left\|\eta_{l}\right\|_{\mathcal{M}} \leq \mathcal{Q}(R)
$$

given by Proposition 4.2. First of all, as

$$
\left|\sigma\left(\partial_{t} u_{1}\right)-\sigma\left(\partial_{t} u_{2}\right)\right| \leq c\left(1+\left|\partial_{t} u_{1}\right|^{4}+\left|\partial_{t} u_{2}\right|^{4}\right)\left|\partial_{t} \bar{u}\right|,
$$

we deduce

$$
\begin{aligned}
-\left\langle\sigma\left(\partial_{t} u_{1}\right)-\sigma\left(\partial_{t} u_{2}\right), \partial_{t} \bar{w}\right\rangle & \leq c\left(1+\left\|\partial_{t} u_{1}\right\|_{L^{6}}^{4}+\left\|\partial_{t} u_{2}\right\|_{L^{6}}^{4}\right)\left\|\partial_{t t} \bar{w}\right\|_{L^{6}}\left\|\partial_{t} \bar{w}\right\|_{L^{6}} \\
& \leq c\left(1+\left\|\partial_{t} u_{1}\right\|_{1}^{4}+\left\|\partial_{t} u_{2}\right\|_{1}^{4}\right)\left\|\partial_{t t} \bar{w}\right\|_{1}\left\|\partial_{t} \bar{w}\right\|_{1} \\
& \leq \mathcal{Q}(R)\left\|\partial_{t} \bar{w}\right\|_{1}^{2}+\frac{1}{2}\left\|\partial_{t t} \bar{w}\right\|_{1}^{2}
\end{aligned}
$$


Besides, we have

$$
\begin{aligned}
-\gamma\left\langle\partial_{t} \bar{w}, \partial_{t t} \bar{w}\right\rangle_{\theta}-\left\langle\bar{w}, \partial_{t t} \bar{w}\right\rangle_{1}-\int_{0}^{\infty} \mu(s)\left\langle\bar{\xi}(s), \partial_{t t} \bar{w}\right\rangle_{1} \mathrm{~d} s & \leq\left(\|\bar{w}\|_{1}+\gamma\left\|\partial_{t} \bar{w}\right\|_{2 \theta-1}+\sqrt{\kappa}\|\bar{\xi}\|_{\mathcal{M}}\right)\left\|\partial_{t t} \bar{w}\right\|_{1} \\
& \leq c\left(\|\bar{w}\|_{1}^{2}+\left\|\partial_{t} \bar{w}\right\|_{1}^{2}+\|\bar{\xi}\|_{\mathcal{M}}^{2}\right)+\frac{1}{2}\left\|\partial_{t t} \bar{w}\right\|_{1}^{2} .
\end{aligned}
$$

Hence, owing to the estimate

$$
\left\langle\bar{\eta}^{0}-\bar{u}(0), \bar{\xi}\right\rangle_{\mathcal{M}} \leq\|\bar{u}(0)\|_{1}^{2}+\left\|\bar{\eta}^{0}\right\|_{\mathcal{M}}^{2}+c\|\bar{\xi}\|_{\mathcal{M}}^{2},
$$

the differential inequality above becomes

$$
\frac{\mathrm{d}}{\mathrm{d} t} \Lambda \leq Q \mathcal{Q}(R) \Lambda-\frac{\mathrm{d}}{\mathrm{d} t}\left\langle F, \bar{w}+\partial_{t} \bar{w}\right\rangle+\frac{\mathrm{d}}{\mathrm{d} t}\left\langle G, \bar{w}+\partial_{t} \bar{w}\right\rangle+\left\langle\partial_{t} F, \bar{w}+\partial_{t} \bar{w}\right\rangle+\|\bar{u}(0)\|_{1}^{2}+\left\|\bar{\eta}^{0}\right\|_{\mathcal{M}}^{2} .
$$

At this point, we choose an arbitrary $x \in[0, \tau]$, and we integrate the latter inequality on $(0, x)$. This yields

$$
\begin{aligned}
\Lambda(x) \leq & \mathcal{Q}(R) \int_{0}^{x} \Lambda(t) \mathrm{d} t-\left\langle F(x), \bar{w}(x)+\partial_{t} \bar{w}(x)\right\rangle+\left\langle G, \bar{w}(x)+\partial_{t} \bar{w}(x)\right\rangle-\langle G, \bar{u}(0)\rangle \\
& +\int_{0}^{x}\left\langle\partial_{t} F(t), \bar{w}(t)+\partial_{t} \bar{w}(t)\right\rangle \mathrm{d} t+(1+x)\|\bar{u}(0)\|_{1}^{2}+x\left\|\bar{\eta}^{0}\right\|_{\mathcal{M}}^{2} .
\end{aligned}
$$

Since

$$
-\left\langle F(x), \bar{w}(x)+\partial_{t} \bar{w}(x)\right\rangle+\left\langle G, \bar{w}(x)+\partial_{t} \bar{w}(x)\right\rangle-\langle G, \bar{u}(0)\rangle \leq c\|F(x)\|_{-1}^{2}+c\|G\|_{-1}^{2}+\frac{1}{2} \Lambda(x)+c\|\bar{u}(0)\|_{1}^{2},
$$

we get

$$
\Lambda(x) \leq \mathcal{Q}(R) \int_{0}^{x} \Lambda(t) \mathrm{d} t+c\|F(x)\|_{-1}^{2}+c\|G\|_{-1}^{2}+\int_{0}^{x}\left\langle\partial_{t} F(t), \bar{w}(t)+\partial_{t} \bar{w}(t)\right\rangle \mathrm{d} t+c(1+x)\left\|z_{1}-z_{2}\right\|_{\mathcal{H}}^{2} .
$$

Due to (1.3),

$$
\begin{aligned}
\|F(x)\|_{-1}^{\frac{6}{5}} & \leq c\|F(x)\|_{L^{6 / 5}}^{\frac{6}{5}} \\
& \leq c x^{\frac{1}{5}} \int_{0}^{x} \int_{\Omega}\left(1+\left|u_{1}(t)\right|^{4}+\left|u_{2}(t)\right|^{4}\right)^{\frac{6}{5}}|\bar{u}(t)|^{\frac{6}{5}} \mathrm{~d} \boldsymbol{x} \mathrm{d} t \\
& \leq c x^{\frac{1}{5}} \int_{0}^{x}\left(\int_{\Omega}\left(1+\left|u_{1}(t)\right|^{4}+\left|u_{2}(t)\right|^{4}\right)^{\frac{3}{2}} \mathrm{~d} \boldsymbol{x}\right)^{\frac{4}{5}}\|\bar{u}(t)\|_{L^{6}}^{\frac{6}{5}} \mathrm{~d} t \\
& \leq x^{\frac{1}{5}} Q(R) \int_{0}^{x}\|\bar{u}(t)\|_{1}^{\frac{6}{5}} \mathrm{~d} t,
\end{aligned}
$$

implying

$$
\|F(x)\|_{-1}^{2} \leq x^{\frac{1}{3}} \mathcal{Q}(R)\left(\int_{0}^{x}\|\bar{u}(t)\|_{1}^{\frac{6}{5}} \mathrm{~d} t\right)^{\frac{5}{3}} \leq x \mathcal{Q}(R) \int_{0}^{x}\|\bar{u}(t)\|_{1}^{2} \mathrm{~d} t \leq x \mathcal{Q}(R) \int_{0}^{x} \Lambda(t) \mathrm{d} t .
$$

Analogously, by (5.5),

$$
\begin{aligned}
\left\|\sigma\left(\partial_{t} u_{1}(0)\right)-\sigma\left(\partial_{t} u_{2}(0)\right)\right\|_{L^{6 / 5}}^{\frac{6}{5}} & \leq c \int_{\Omega}\left(1+\left|\partial_{t} u_{1}(0)\right|^{4}+\left|\partial_{t} u_{2}(0)\right|^{4}\right)^{\frac{6}{5}}\left|\partial_{t} \bar{u}(0)\right|^{\frac{6}{5}} \mathrm{~d} \boldsymbol{x} \\
& \leq \mathcal{Q}(R)\left\|\partial_{t} \bar{u}(0)\right\|_{1}^{\frac{6}{5}},
\end{aligned}
$$

which yields

$$
\|G\|_{-1}^{2} \leq c\|\bar{u}(0)\|_{1}^{2}+Q(R)\left\|\partial_{t} \bar{u}(0)\right\|_{1}^{2} .
$$

Reasoning as before, we find

$$
\left\|\partial_{t} F\right\|_{L^{6 / 5}}^{\frac{6}{5}} \leq c \int_{\Omega}\left(1+\left|u_{1}\right|^{4}+\left|u_{2}\right|^{4}\right)^{\frac{6}{5}}|\bar{u}|^{\frac{6}{5}} \mathrm{~d} \boldsymbol{x} \leq \mathcal{Q}(R)\|\bar{u}\|_{1}^{\frac{6}{5}},
$$


whence

$$
\begin{aligned}
\int_{0}^{x}\left\langle\partial_{t} F(t), \bar{w}(t)+\partial_{t} \bar{w}(t)\right\rangle \mathrm{d} t & \leq \int_{0}^{x}\left\|\partial_{t} F(t)\right\|_{L^{6 / 5}}\left(\|\bar{w}(t)\|_{L^{6}}+\left\|\partial_{t} \bar{w}(t)\right\|_{L^{6}}\right) \mathrm{d} t \\
& \leq \mathcal{Q}(R) \int_{0}^{x} \Lambda(t) \mathrm{d} t .
\end{aligned}
$$

In light of the above computations, (5.7) improves to

$$
\Lambda(x) \leq(1+x) \mathcal{Q}(R) \int_{0}^{x} \Lambda(t) \mathrm{d} t+(1+x) \mathcal{Q}(R)\left\|z_{1}-z_{2}\right\|_{\mathcal{H}}^{2}
$$

for every $x \in[0, \tau]$, and an application of the Gronwall lemma entails

$$
\Lambda(x) \leq(1+\tau) \mathcal{Q}(R) e^{\tau(1+\tau) \mathcal{Q}(R)}\left\|z_{1}-z_{2}\right\|_{\mathscr{H}}^{2}, \quad \forall x \in[0, \tau] .
$$

In particular we learn that

$$
\|\bar{u}(x)\|_{1}^{2} \leq(1+\tau) \mathcal{Q}(R) e^{\tau(1+\tau) Q(R)}\left\|z_{1}-z_{2}\right\|_{\mathscr{H}}^{2}, \quad \forall x \in[0, \tau] .
$$

The analogous estimate for $\partial_{t} \bar{u}$ comes directly from (5.3). Indeed, taking into account (5.6), (5.8) and the controls for $\|F\|_{-1}$ and $\|G\|_{-1}$ obtained before,

$$
\left\|\partial_{t} \bar{u}\right\|_{1}^{2} \leq \frac{1}{2}\left(\gamma\left\|\partial_{t} \bar{w}\right\|_{2 \theta-1}+\|\bar{w}\|_{1}+\sqrt{\kappa}\|\bar{\xi}\|_{\mathcal{M}}+\|F\|_{-1}+\|G\|_{-1}\right)^{2}+\frac{1}{2}\left\|\partial_{t} \bar{u}\right\|_{1}^{2},
$$

and we draw the estimate

$$
\left\|\partial_{t} \bar{u}(x)\right\|_{1}^{2} \leq\left(1+\tau^{3}\right) \mathcal{Q}(R) e^{\tau(1+\tau) \mathcal{Q}(R)}\left\|z_{1}-z_{2}\right\|_{\mathscr{H}}^{2}, \quad \forall x \in[0, \tau] .
$$

Finally, to control $\|\bar{\eta}\|_{\mathcal{M}}$, we multiply the equation for $\bar{\eta}$, i.e.

$$
\partial_{t} \bar{\eta}=T \bar{\eta}+\partial_{t} \bar{u}
$$

by $\bar{\eta}$ in $\mathcal{M}$, to get

$$
\frac{\mathrm{d}}{\mathrm{d} t}\|\bar{\eta}\|_{\mathcal{M}}^{2}=\langle T \bar{\eta}, \bar{\eta}\rangle_{\mathcal{M}}+\left\langle\partial_{t} \bar{u}, \bar{\eta}\right\rangle_{\mathcal{M}} \leq \sqrt{\kappa}\left\|\partial_{t} \bar{u}\right\|_{1}\|\bar{\eta}\|_{\mathcal{M}} \leq\|\bar{\eta}\|_{\mathcal{M}}^{2}+c\left\|\partial_{t} \bar{u}\right\|_{1}^{2} .
$$

By the Gronwall lemma, exploiting the estimate for $\left\|\partial_{t} \bar{u}\right\|_{1}$ just proved, we are led to

$$
\left\|\bar{\eta}^{x}\right\|_{\mathcal{M}}^{2} \leq\left(1+\tau^{3}\right) \mathcal{Q}(R) e^{\tau(1+\tau) \mathcal{Q}(R)}\left\|z_{1}-z_{2}\right\|_{\mathscr{H}}^{2}, \quad \forall x \in[0, \tau] .
$$

The proof is finished.

\section{The solution semigroup}

For any given initial datum $z \in \mathcal{H}$, we now write the unique solution $\left(u(t), \eta^{t}\right)$ to (3.1) at time $t>0$ with initial conditions

$$
\left(u(0), \partial_{t} u(0), \eta^{0}\right)=z
$$

in the form

$$
\left(u(t), \partial_{t} u(t), \eta^{t}\right)=S(t) z
$$

where the map $S(t): \mathscr{H} \rightarrow \mathscr{H}$ is a semigroup of operators, namely,

- $S(0)=\operatorname{id}_{\mathscr{H}}$ (the identity map in $\mathscr{H}$ ),

- $S(t+\tau)=S(t) S(\tau)$ for all $t, \tau \geq 0$.

Collecting Theorems 4.1 and 5.1, we can formulate the following result.

Theorem 6.1. The semigroup $S(t)$ satisfies the strong continuity property

$$
z \mapsto S(t) z \in \mathcal{C}(\mathcal{H}, \mathcal{H}), \quad \forall t \geq 0 .
$$

Besides, $S(t)$ fulfills the further continuity in time

$$
t \mapsto S(t) z \in \mathcal{C}([0, \infty), \mathscr{H}), \quad \forall z \in \mathcal{H} .
$$


Remark 6.2. Actually, it is easily seen that $S(t)$ is jointly continuous, that is,

$$
(t, z) \mapsto S(t) z \in \mathcal{C}([0, \infty) \times \mathscr{H}, \mathcal{H}) .
$$

In addition, by the a priori estimates of Proposition 4.2 we also learn that the solution fulfills

$$
u \in W^{2, \infty}\left(0, \infty ; H^{1}\right), \quad \eta \in L^{\infty}(0, \infty ; \mathcal{M})
$$

and, if $\gamma>0$,

$\partial_{t} u \in L^{2}\left(0, \infty ; H^{\theta}\right)$.

The latter relation is an immediate consequence of (4.1)-(4.2).

\section{References}

[1] A.H. Love, A Treatise on Mathematical Theory of Elasticity, Dover, New York, 1944.

[2] M.M. Cavalcanti, V.N. Domingos Cavalcanti, J. Ferreira, Existence and uniform decay for a non-linear viscoelastic equation with strong damping, Math. Methods Appl. Sci. 24 (2001) 1043-1053.

[3] X. Han, M. Wang, General decay of energy for a viscoelastic equation with nonlinear damping, Math. Methods Appl. Sci. 32 (2009) $346-358$.

[4] X. Han, M. Wang, Global existence and uniform decay for a nonlinear viscoelastic equation with damping, Nonlinear Anal. 70 (2009) $3090-3098$.

[5] W. Liu, Uniform decay of solutions for a quasilinear system of viscoelastic equations, Nonlinear Anal. 71 (2009) 2257-2267.

[6] W. Liu, General decay and blow-up of solution for a quasilinear viscoelastic problem with nonlinear source, Nonlinear Anal. 73 (2010) 1890-1904.

[7] S.A. Messaoudi, M.I. Mustafa, A general stability result for a quasilinear wave equation with memory, Nonlinear Anal. RWA 14 (2013) $1854-1864$.

[8] S.A. Messaoudi, N.-E. Tatar, Global existence and uniform stability of solutions for a quasilinear viscoelastic problem, Math. Methods Appl. Sci. 30 (2007) 665-680.

[9] S.A. Messaoudi, N.-E. Tatar, Exponential and polynomial decay for a quasilinear viscoelastic equation, Nonlinear Anal. 68 (2008) $785-793$.

[10] S.A. Messaoudi, N.-E. Tatar, Exponential decay for a quasilinear viscoelastic equation, Math. Nachr. 282 (2009) 1443-1450.

[11] J.Y. Park, S.H. Park, General decay for quasilinear viscoelastic equations with nonlinear weak damping, J. Math. Phys. 50 (083505) (2009) 10 pp.

[12] S.-T. Wu, Arbitrary decays for a viscoelastic equation, Bound. Value Probl. 28 (2011) 14 pp.

[13] R.O. Araujo, T.F. Ma, Y. Qin, Long-time behavior of a quasilinear viscoelastic equation with past history, J. Differential Equations 254 (2013) $4066-4087$.

[14] M. Grasselli, V. Pata, Uniform attractors of nonautonomous dynamical systems with memory, in: A. Lorenzi, B. Ruf (Eds.), Evolution Equations, Semigroups and Functional Analysis, in: Progr. Nonlinear Differential Equations Appl., No. 50, Birkhäuser, Basel, 2002, pp. 155-178.

[15] C.M. Dafermos, Asymptotic stability in viscoelasticity, Arch. Ration. Mech. Anal. 37 (1970) 297-308.

[16] A. Pazy, Semigroups of Linear Operators and Applications to Partial Differential Equations, Springer-Verlag, New York, 1983.

[17] M. Conti, S. Gatti, V. Pata, Uniform decay properties of linear Volterra integro-differential equations, Math. Models Methods Appl. Sci. 18 (2008) 21-45.

[18] C. Giorgi, V. Pata, A. Marzocchi, Asymptotic behavior of a semilinear problem in heat conduction with memory, NoDEA Nonlinear Differential Equations Appl. 5 (1998) 333-354. 